\title{
Shape Optimization of Mechanical Components for Measurement Systems
}

\author{
Alexander Janushevskis, Janis Auzins, \\ Anatoly Melnikovs and Anita Gerina-Ancane \\ Riga Technical University, \\ Latvia
}

\section{Introduction}

For the topology and shape optimization of structures, the different realizations of homogenization method are widely used (Arora, 2004; Bendsoe \& Sigmund, 2003). This method is highly effective for shell constructions and allows implementing topometry and topography, sizing and shape as well as freeform optimization (Vanderplaats, 2004). However, it is a very time consuming procedure because the number of design parameters can reach a million and more. There is the possibility of taking into account some technological factors, nevertheless, in the case of bulky bodies it frequently produces shapes that are difficult to manufacture. As shown in work (Mullerschon et al., 2010), the Hybrid Cellular Automata method does not allow parallelization of computations and PBS queuing system has been used. At the same time the following resource saving approach (Janushevskis et al., 2010; Janushevskis \& Melnikovs, 2010) can be used for shape optimization which includes the following steps: 1) Planning the position of control points of NURBS (see, for example, Saxena \& Sahay, 2005) for obtaining a smooth shape. 2) Building geometrical models using CAD software in conformity with design of experiment. 3) Calculation of responses for a complete FEM model using CAE software. 4) Building metamodels (surrogate models) for responses on the basis of computer experiment. 5) Using metamodels for shape optimization. 6) Validating the optimal design using CAE software for the complete FEM model.

\section{Basics of approach}

Latin Hypercube type experimental designs first proposed by Vilnis Eglajs in his work (Audze \& Eglajs, 1977), then by McKay (McKay et al., 1979) and used by many other investigators, as well as its improvements (Auzins, 2004) are a very essential aspect utilized in the proposed method. The significance of approximations for the solution of optimization problems proposed by Lucien Schmit in his early works (Schmit \& Farcshi, 1971) and nowadays generally recognized as the Response Surface Method is also the foundation of current approach. The use of planned computer experiments and the metamodeling (surrogate model) approach (Forrester et al., 2008; Sacks et al., 1989) ensures great economy of computing time, especially for finite element (FEM) calculations. First of all let us demonstrate our approach on the simple test problems. 


\subsection{Test problem of plate bending}

A clamped square plate is considered under a concentrated load of $500 \mathrm{~N}$ applied at centre in a direction orthogonal to its main surface. The isotropic material properties are: the Young's modulus $E=200 \mathrm{GPa}$, the Poisson's ratio $v=0.3$ and dimensions are $400 \times 400 \times 4.2118 \mathrm{~mm}$. The shape optimization of the plate with constant thickness is carried out to minimize its volume in the case of a single displacement constraint $\delta=0.5 \mathrm{~mm}$. The cutout shape of the plate is defined by subsequent techniques shown in Figure 1: 1) with the points that are connected with straight lines; 2) with the NURBS knot points; 3) with the control points of NURBS polygon. Due to symmetry only $1 / 8$ of the plate is considered for cutout definition and $1 / 4$ of the plate for problem solution by FEM.

1)

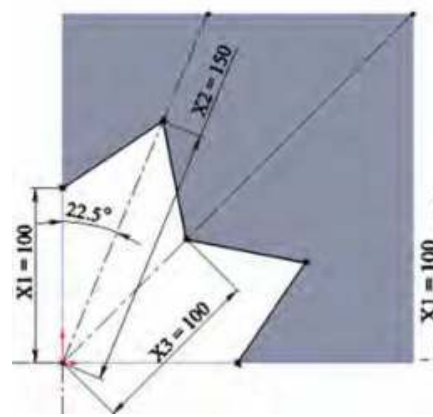

2)

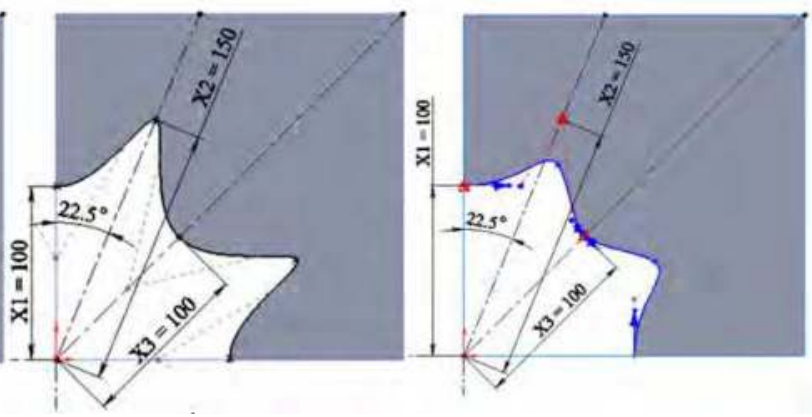

Fig. 1. Techniques for definition of cutout.

Three parameters are stated to define location of points. Parameters are varied in the following ranges: $100 \leq X 1 \leq 170 ; 100 \leq X 2 \leq 210 ; 100 \leq X 3 \leq 230 \mathrm{~mm}$ for the first two variants and $100 \leq X 1 \leq 180 ; 100 \leq X 2 \leq 235 ; 100 \leq X 3 \leq 230 \mathrm{~mm}$ for third variant of definition. In the last case at both end points two continuity vectors are defined additionally with direction normal to the side and to symmetry axis of the corresponding plate and with fixed length of 19 and $3 \mathrm{~mm}$. The design of experiment for 3 factors and 40 trial points is calculated with mean-square error (MSE) criterion (Auzins \& Janushevskis, 2002; Auzins, et al., 2006) value 0.4262 using EDAOpt (Auzins \& Janushevskis, 2007) - software for design of experiments, approximation and optimization developed in the Riga Technical University. The geometrical models are developed using SolidWorks (SW) for all variants. The shapes for the third variant of definition are shown in Figure 2. In the next step responses of these models are calculated by SW Simulation (Lombard, 2009), using elements with a global size $4 \mathrm{~mm}$ and total number of DoF $\sim 100000$. Then these responses are used for approximation by EDAOpt. For example, for approximation of response $y$ by quadratic polynomial the following expression (see, for example, Auzins \& Janushevskis, 2007) is used:

$$
\hat{y}=\beta_{0}+\sum_{i=1}^{d} \beta_{i} x_{i}+\sum_{i=1}^{d-1} \sum_{j=i+1}^{d} \beta_{i j} x_{i} x_{j}+\sum_{i=1}^{d} \beta_{i j} x_{i}^{2}+\varepsilon
$$

where there are $d$ variables $x_{1}, \ldots, x_{d}, L=(d+1)(d+2) / 2$ unknown coefficients $\beta$ and the errors $\varepsilon$ are assumed independent with zero mean and constant variance $\sigma^{2}$. 

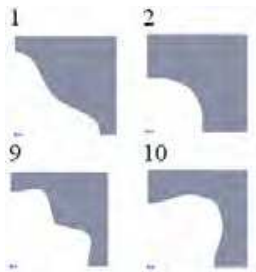

10
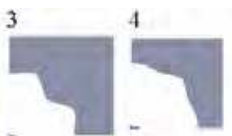

11

12

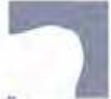

17

18

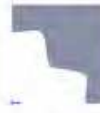

19

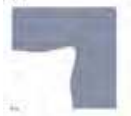

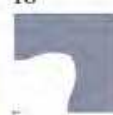

25

26
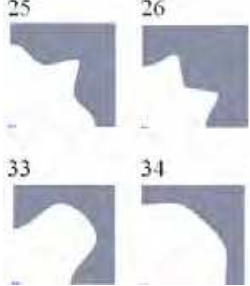

34

35
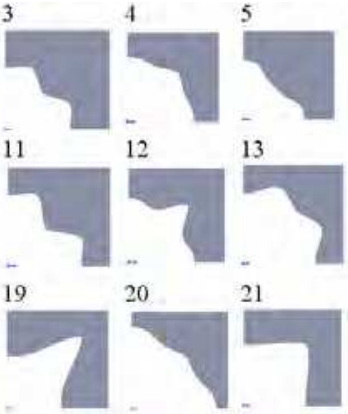

6

13

20

27
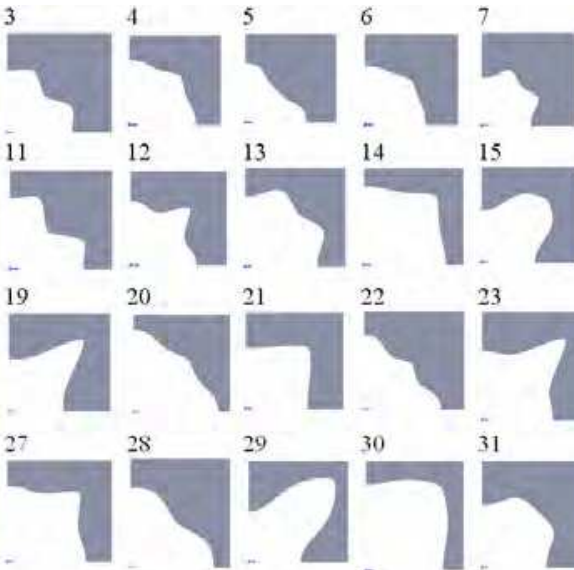

14

15

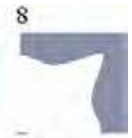

16
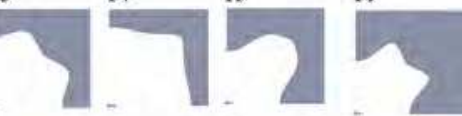

21

22

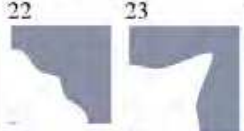

24
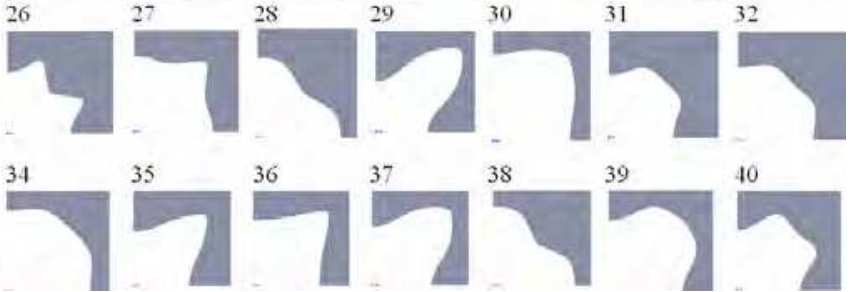

40

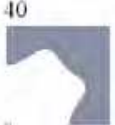

Fig. 2. Shapes of cutout in compliance with design of experiment.

In the case of locally weighted polynomial approximation, coefficients $\beta=\left(\beta_{1}, \beta_{2}, \beta_{3}, \ldots, \beta_{\mathrm{L}}\right)$ depend on point $x_{0}$ where prediction is calculated and are obtained using the weighted least squares method:

$$
\beta=\arg \min _{\beta} \sum_{j \in N_{X}} w\left(x_{0}-x_{j}\right) \times\left(y_{j}-\hat{y}\left(x_{j}\right)\right)^{2}
$$

where the significance of neighboring points in the set $N_{X}$ is taken into account by Gaussian kernel (weight function):

$$
w(u)=\exp \left(-\alpha u^{2}\right)
$$

where $u$ is Euclidian distance from $x_{0}$ to current point and $\alpha$ is a coefficient that characterizes significance.

Quality of approximation is estimated by relative crossvalidation error using leave-one-out crossvalidation:

$$
\sigma_{e r r}=100 \% \frac{\sqrt{\frac{1}{n} \sum_{i=1}^{n}\left(\hat{y}_{-i}\left(x_{i}\right)-y_{i}\right)^{2}}}{\sqrt{\frac{1}{n-1} \sum_{i=1}^{n}\left(y_{i}-\bar{y}\right)^{2}}}
$$

where root mean squared prediction error stands in numerator and mean square deviation of response from its average value stands in denominator, $n$ is the total number of 
experimental trials and $\hat{y}_{-i}\left(x_{i}\right)$ denotes approximated value for response in $i$-th point, calculated without taking into account the $i$-th experimental point.

Using the obtained locally weighted polynomial approximations by global search procedure (Janushevskis et. al, 2004), implemented in EDAOpt, the optimal cutout shape is obtained (see Figure 3) for the different aforementioned techniques. In table 1 the results are summarized and compared with volume obtained in work (Liang et al., 2001) using the homogenization method. Variants correspond to shapes shown in figure 3. The value of Gaussian kernel parameter $\alpha$ of the local quadratic polynomial approximation is chosen to minimize relative leave-one-out crossvalidation error $\sigma_{e r r}$ of approximations of appropriate responses, i.e. deflection $\delta$ and volume $v$ of plate. $v_{p}$ is the predicted volume calculated using approximations and $v_{a}$ is the actual volume calculated using a geometrical model. $v_{p}$ and $v_{a}$ in \% show comparison of appropriate volume in respect to the volume obtained in (Liang et al., 2001). Best results are achieved with the technique using the control points of NURBS polygon. This allows reducing the volume of the plate by $1.38 \%$ (Fig. 3 e) in comparison with the homogenization method.

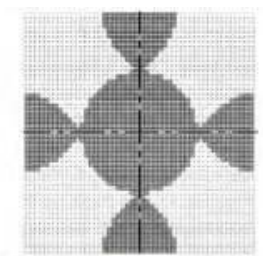

a)

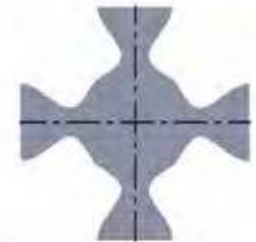

d)

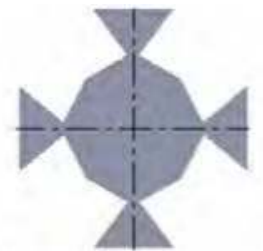

b)

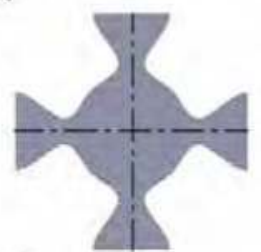

e)

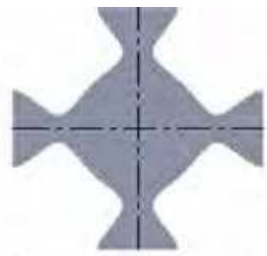

c)

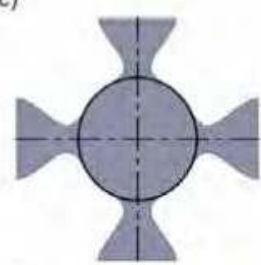

f)

Fig. 3. Shapes of plate obtained by (a) homogenization method (Liang et al., 2001); and by the current approach using different techniques: (b) the points that are connected with straight lines; (c) with the NURBS knot points; (d) with the control points of NURBS polygon; (e) same as " $\mathrm{d}$ " but with additionally optimized tangent weighting at the spline endpoints; (f) same as " $\mathrm{e}$ " but with circle added.

\begin{tabular}{cccccccc}
\hline Variant & $\alpha$ & $\begin{array}{c}\sigma_{\text {err } \delta} \\
\%\end{array}$ & $\begin{array}{c}\sigma_{\text {err }} \\
\%\end{array}$ & $\begin{array}{c}v_{p} \\
m m^{3}\end{array}$ & $\begin{array}{c}v_{a} \\
m m^{3}\end{array}$ & $\begin{array}{c}v_{p} \\
\%\end{array}$ & $\begin{array}{c}v_{a} \\
\%\end{array}$ \\
\hline $\mathrm{a}$ & - & - & - & - & 68750.00 & - & - \\
$\mathrm{b}$ & 17 & 9.81 & 0.03 & 69414.78 & 69331.58 & 1 & 0.84 \\
$\mathrm{c}$ & 17 & 9.81 & 0.03 & 68988.67 & 68815.88 & 0.4 & 0.096 \\
$\mathrm{~d}$ & 15.6 & 9.81 & 0.03 & 68862.32 & 68721.98 & 0.16 & -0.04 \\
$\mathrm{e}$ & 3.2 & 0.79 & 0.16 & 67797.524 & 67800.975 & -1.385 & -1.38 \\
\hline
\end{tabular}

Table 1. Quantitative indices of the shape optimization of cutout for the plate bending problem. 
It should be mentioned that the predicted volume for variant e is in good agreement with the actual value. At the same time the total number of FEM problem calculations (i.e. number of trials of computer experiment) is less on 10 than by using the homogenization method. The distribution of resultant displacement for plate of optimal shape (variant e) is shown in fig. 4.

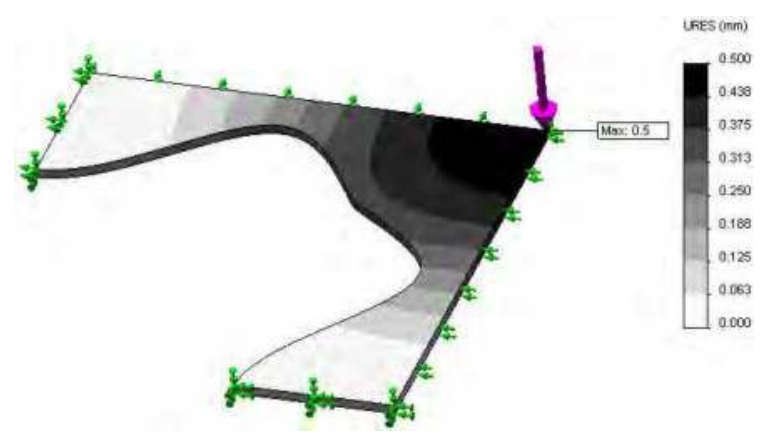

Fig. 4. Displacement of plate for variant e.

\subsection{Stretched plate test problem}

A square plate with dimensions $1300 \times 1300 \times 0.0001 \mathrm{~mm}$ is considered under two axial stretching loads of $\mathrm{p}=0.65 \mathrm{~N} / \mathrm{mm}^{2}$ and $\mathrm{p} / 2$ accordingly (see Figure 6). The isotropic material properties are: the Young's modulus $E=210 \mathrm{GPa}$, the Poisson's ratio $v=0.3$. The shape optimization of the plate with constant thickness is carried out to minimize its volume in case of a single constraint on maximal level of equivalent stress $\sigma_{\max }=4.38 \mathrm{MPa}$. Due to symmetry only $1 / 4$ of the plate is considered for cutout definition and for problem solution by FEM. The cutout shape of the plate is defined by five coordinates of points 2, 3, 4, 5 and 6 situated on straight lines that make angles of $0 ; 22.5 ; 45$ and 90 degrees with horizontal axis as shown in Figure 5. The initial cutout shape is a circle with radius $250 \mathrm{~mm}$ and the volume of plate $v$ is $373.4 \mathrm{~mm}^{3}$.

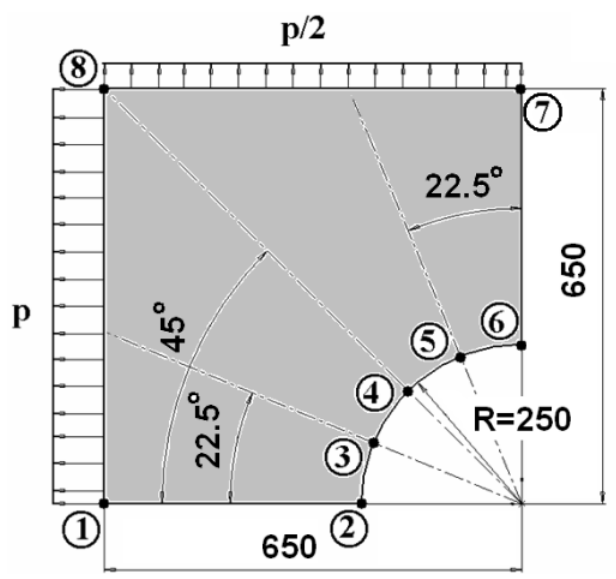

Fig. 5. Scheme of $1 / 4$ of plate with initial cutout. 
The aforementioned five parameters are varied in the range from 250 to $640 \mathrm{~mm}$. The design of experiment for 5 factors and 112 trial points is calculated with MSE criterion value 0.5394. In the next step responses of these models are calculated by SW Simulation using shell elements with global size $7.5 \mathrm{~mm}$ and total number of DoF 108000 . Then these responses are used for approximation by EDAOpt. Using obtained locally weighted polynomial approximations by global search procedure, the optimal cutout shape is obtained (see Figure 6) for the different aforementioned techniques. In table 2 the results are summarized and compared with the volume obtained in work (Papadrakakis et al., 1998). Variants correspond to shapes shown in figure 6.

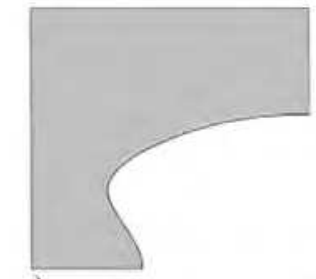

a)

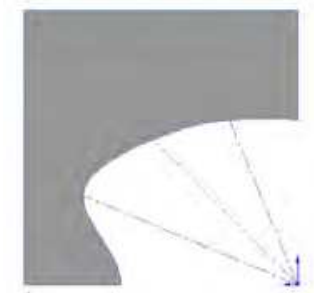

c)

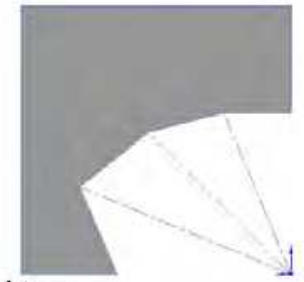

b)

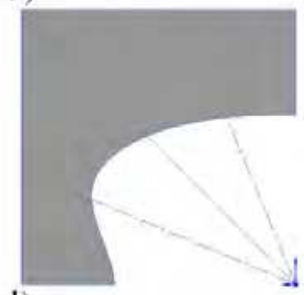

d)

Fig. 6. The obtained plate shapes. Plate shapes obtained (a) in (Papadrakakis et al., 1998); and by current approach using different techniques: (b) the points that are connected with straight lines; (c) with the NURBS knot points; (d) with the control points of NURBS polygon.

\begin{tabular}{cccccccc}
\hline Variant & $\alpha$ & $\begin{array}{c}\sigma_{\text {err }} \sigma \\
\%\end{array}$ & $\begin{array}{c}\sigma_{\text {err }} \\
\%\end{array}$ & $\begin{array}{c}v_{p} \\
m m^{3}\end{array}$ & $\begin{array}{c}v_{a} \\
m m^{3}\end{array}$ & $\begin{array}{c}\sigma_{\max } \\
M P a\end{array}$ & $\begin{array}{c}\sigma_{\max a} \\
M P a\end{array}$ \\
\hline $\mathrm{a}$ & - & & - & - & 280 & - & 4.38 \\
$\mathrm{~b}$ & 8 & 48.73 & 0.00 & 255.93 & 255.84 & 4.38 & 5.22 \\
$\mathrm{c}$ & 12.6 & 47.48 & 2.27 & 251.76 & 251.38 & 4.38 & 4.35 \\
$\mathrm{~d}$ & 7.3 & 31.94 & 1.17 & 251.00 & 250.69 & 4.38 & 4.34 \\
& & & & & & & \\
\hline
\end{tabular}

Table 2. Quantitative indices of the shape optimization of cutout for the plate stretching problem.

The value of Gaussian kernel parameter $\alpha$ of the local quadratic polynomial approximation is chosen to minimize relative leave-one-out crossvalidation error $\sigma_{\text {err }}$ of approximations of appropriate responses, i.e. maximal equivalent stress $\sigma_{\max }$ and volume $v$ of plate. $v_{p}$ is predicted volume calculated using approximations and $v_{a}$ is actual volume calculated using full FEM and geometrical models. Again the best results are achieved with the technique using the control points of NURBS polygon. This allows reducing volume of the plate by 
$10.5 \%$ (Fig. 6 d) in comparison with the volume obtained in (Papadrakakis et al., 1998). Distribution of equivalent von Mises stress for case $d$ is shown in Figure 7.

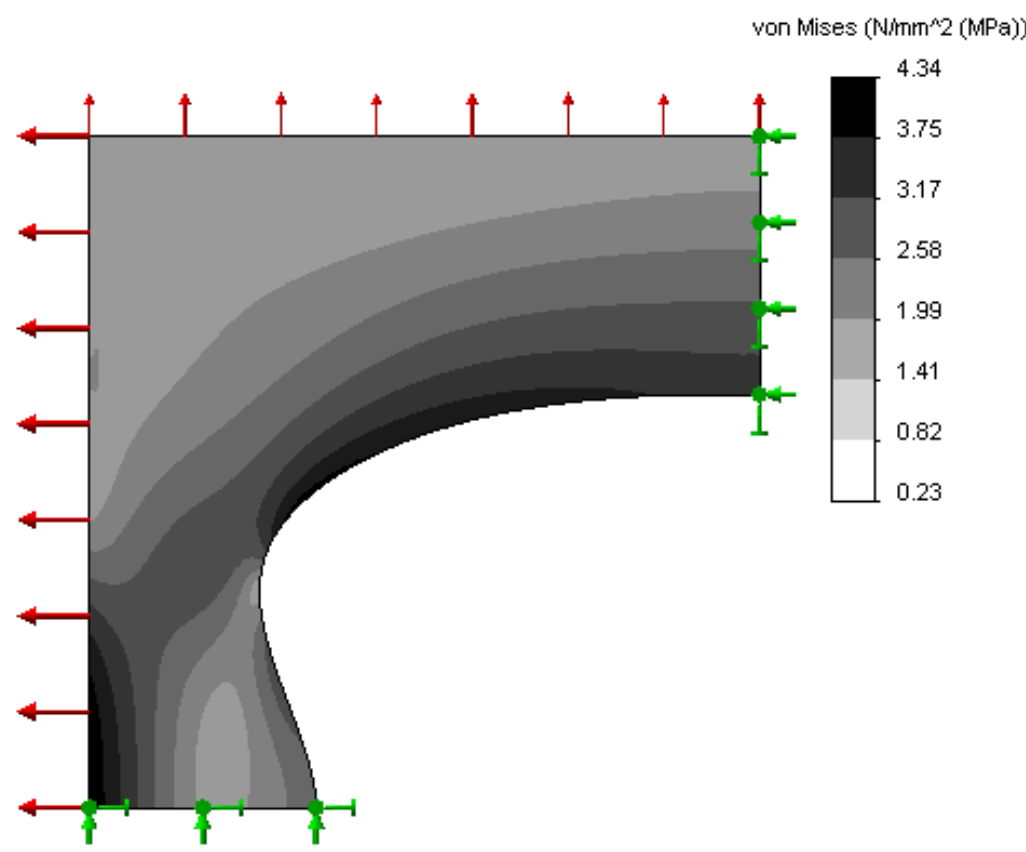

Fig. 7. Equivalent von Mises stress levels in the plate for variant $d$.

\section{Tensometric wheel pairs}

At the present time special tensometric wheel pairs are used for the wheel - rail system monitoring. For each type of rolling stock these wheel pairs must fit the vehicle's wheel wearing condition, diameter and bearing box connection type. Using and delivering the tensometric wheel pairs is expensive and takes a lot of time for preparing strength dynamics tests. In this work removable equipment for monitoring is proposed for mounting on the ordinary wheel pairs. The monitoring wireless system (Grigorov, 2004; Hart, 1986) for 80 tons wagon (freight car) is taken for prototype. The movable part of the equipment (Fig. 8) consists of a removable disk, two transmitters and a transmitting antenna as well as strain gauges bonded to the wheel at defined places. The removable disk is fixedly attached to the wheel pair's axis. A circular transmitting antenna and two transmitters are mounted on the outside of the disk.

Removable equipment must be lightweight to minimize distortions of measurements and at the same time it must be with appropriate durability. During testing dynamical loads caused by rail joints, railroad switches and other irregularities as well as due to defects of wheel geometry are transmitted from wheel pairs to the removable disk which is rigidly mounted on the wheelset axis. Therefore shape optimization of the mounting disk that is the main heavy-weight part of the equipment is very important to reduce its total weight. 


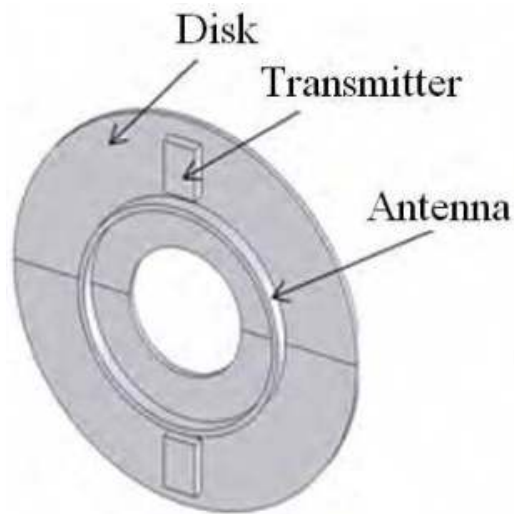

(a)

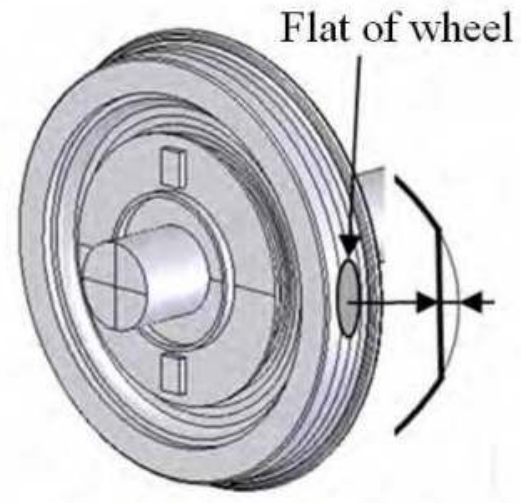

(b)

Fig. 8. (a) Removable disc with elements of measurement system and (b) its mounting place.

\subsection{Loads acting on wheel pairs}

The main loads are acting in vertical direction and are caused by railroad irregularities and wheel defects in the wheel - railroad contact. The removable disk sustains all loads from the wheel pair because it is rigidly fastened. Strength of the removable disk is calculated for maximal possible loading. For example, in the case when the wheel pair has $2 \mathrm{~mm}$ flat of wheel (Fig. 8 b), the loaded and empty wagon wheelsets undergo different loads in vertical direction at different velocities (see Table. 3) (Sladkowsky \& Pogorelov, 2008). For an empty wagon the maximal load is at velocity $5 \mathrm{~m} / \mathrm{s}$, but for the loaded wagon at $10 \mathrm{~m} / \mathrm{s}$. Strength of the removable disk will be analyzed with maximal vertical load $-620.6 \mathrm{kN}$ for two cases of orientation of the transmitters - horizontal and vertical when the wheel pair acceleration can reach $12 \mathrm{~g}$ (the gravitational acceleration $g=9.81 \mathrm{~m} / \mathrm{s}^{2}$ is taken into account).

\begin{tabular}{|c|c|c|}
\hline \multirow{2}{*}{ Velocity of wagon m/s } & \multicolumn{2}{|c|}{ Maximal load in moment of shock, $\mathrm{kN}$} \\
\cline { 2 - 3 } & Empty wagon & Loaded wagon \\
\hline Static load & 22.8 & 104.5 \\
\hline 1 & 136.1 & 251.3 \\
\hline 2 & 170.2 & 316.4 \\
\hline 5 & 297.8 & 367.2 \\
\hline 10 & 271.3 & 620.6 \\
\hline 20 & 276.6 & 604.9 \\
\hline
\end{tabular}

Table 3. Load versus velocity of wagon (Sladkowsky \& Pogorelov, 2008).

Strength of the disk under centrifugal load will also be analyzed at maximum vehicle velocity $200 \mathrm{~km} / \mathrm{h}$ (wheel angular velocity $=116.98 \mathrm{rad} / \mathrm{s}$ ). In this case the disk is considered as new without wear on riding circle.

Besides, frequency analysis was made to find natural frequencies of the wheel pair and evaluate possible resonance in the case of flat of wheel. Obtained results show that excitation frequencies at velocities of operating conditions are significantly smaller than fundamental frequency. 


\subsection{Disk model for strength calculation}

The geometrical model of the disk is created using SW. It takes into account the shape, size and

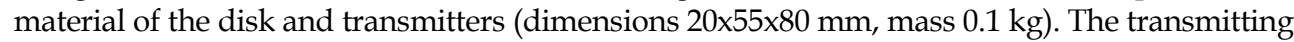
antenna (Fig. 8) is removed from the calculation model because it has small dimensions and lightweight material. The calculation model also doesn't consider fastening holes and fastening elements. The transmitting antenna works stable if its displacement in axial direction is less than $2 \mathrm{~mm}$ (Hart, 1986). This constraint is taken into account in the next optimization.

The strength calculations are performed using SW Simulation. First, the shape of the removable disk (radius $R=300 \mathrm{~mm}$ and thickness $b=10 \mathrm{~mm}$ ) is changed to an ellipse with semi-major axis length $300 \mathrm{~mm}$ and semi-minor axis E1 (Fig. 9 a) by simple size optimization of E1. This shape is convenient for the equipment mounting purposes and is taken for initial design.

FE mesh (Fig. 9 b) is generated with second order tetrahedral solid elements and is generated to get high accuracy results. It consists of about 51000 elements with 86000 nodes (258000 DOF).

Removable disk's displacement is restrained on its cylindrical face (Fig. $9 \mathrm{c}$ ). The material of the disk is aluminum alloy $(1060 \mathrm{H} 12)$ with elastic modulus $E=69000 \mathrm{MPa}$, Poisson's ratio $v=0.33$, mass density $\rho=2700 \mathrm{~kg} / \mathrm{m}^{3}$ and yield strength $\sigma_{y}=27.5742 \mathrm{MPa}$.

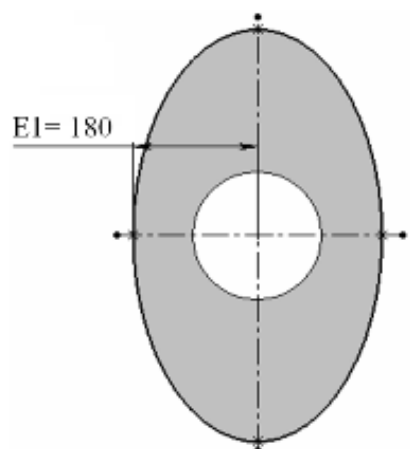

(a)

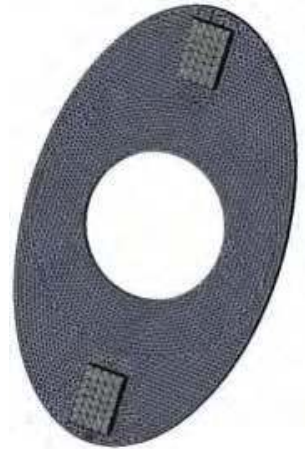

(b)

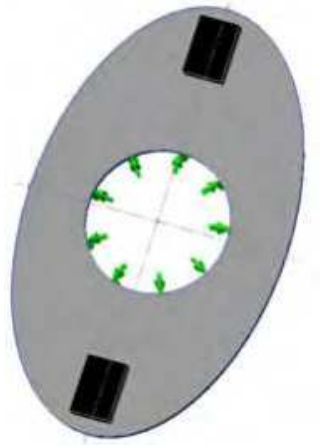

(c)

Fig. 9. (a) Ellipsoidal disk; (b) Computational finite element mesh of the model; (c) Scheme of disk fastening.

The material's ultimate fatigue resistance is calculated as (State Railway Research Institute [SRRI], 1998):

$$
\sigma_{-1}=0.4 \cdot \sigma_{y}
$$

So stresses must be less than $\sigma_{-1}=11.0297 \mathrm{MPa}$. Additionally the value of the factor of safety FOS $=2.75$ is assumed to be sure that the disk will be durable in any worst-case situation (SRRI, 1998). The acceptable stress in the disk material is reduced to $\sigma_{\max }=4 \mathrm{MPa}$. Von Mises yield stress criterion is used for all strength calculations: 


$$
\sigma_{\text {vonMises }}=\sqrt{\frac{\left(\sigma_{1}-\sigma_{2}\right)^{2}+\left(\sigma_{2}-\sigma_{3}\right)^{2}+\left(\sigma_{1}-\sigma_{3}\right)^{2}}{2}},
$$

where $\sigma_{1}, \sigma_{2}, \sigma_{3}$ are principal stresses.

Thereby the von Mises stress at any point of the disk should be less than acceptable stress:

$$
\sigma_{\text {vonMises }}<\sigma_{\max }
$$

\subsection{Stresses in initial design disk}

Three variants of stressed state of the disk are analyzed, i.e., from loads due to flat of wheel in two cases of the disk orientation: when the major axis of ellipse is vertical and horizontal as well as from centrifugal loads.

We consider a loaded wagon with maximal loading in moment of shock that occurs at velocity $10 \mathrm{~m} / \mathrm{s}$ (Table 3). Maximal stresses in moment of shock (acceleration $a=119.3 \mathrm{~m} / \mathrm{s}^{2}$ ) are shown on Fig. 10. As we can see, values of maximal stress levels for both orientations of the disk are very similar.

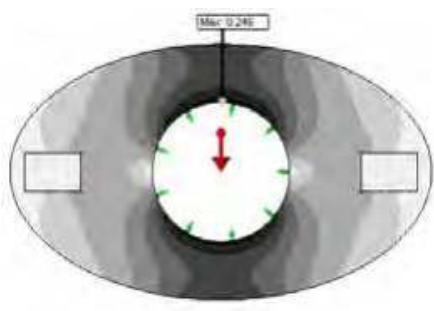

(a)

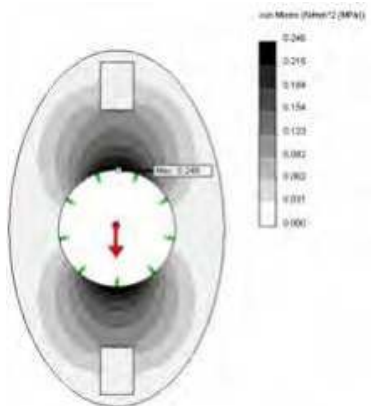

(b)

Fig. 10. Von Mises stresses distribution in initial design disk for (a) horizontal and (b) vertical orientation.

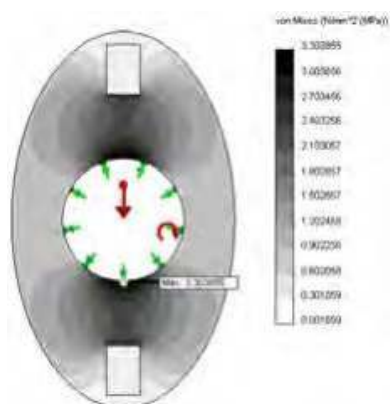

(a)

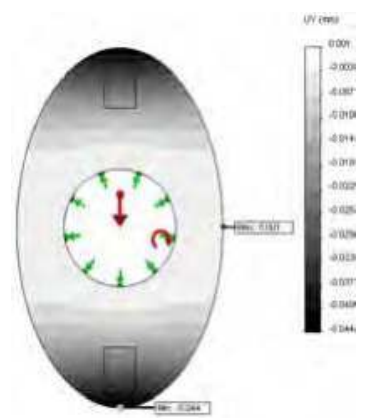

(b)

Fig. 11. (a) Von Mises stresses distribution in initial design disk from centrifugal loads; (b) Disk's displacements in axial direction. 
The results for the stressed state of initial design of the disk from centrifugal load are shown in Fig. 11. Maximum von Mises stress in the disk from centrifugal load is at least 12 times greater than in the case of loading from flat of wheel.

\subsection{Shape optimization of cross section of the ellipsoidal disk}

The shape of cross section of the disk is optimized, taking into account only centrifugal load. The constructive restrictions allow changing the disk cross section shape only at one side and in radial direction at range $150 \mathrm{~mm}$ to $300 \mathrm{~mm}$ from ellipse center. The section of the disk at radial distance $0-150 \mathrm{~mm}$ has constant thickness $\mathrm{b}=10 \mathrm{~mm}$. Three methods are used to define the cross section shape (Fig. 12): a) with NURBS knot points, b) with NURBS polygon points and c) with points that are connected with straight lines. Four parameters are stated to define the shape. Parameters are varied in the following ranges: $4 \leq X 1 \leq 10 ; 4$ $\leq X 2 \leq 10 ; 5 \leq X 3 \leq 12 ; 3 \leq X 4 \leq 5$ for variants "a", "c" and $3 \leq X 1 \leq 10 ; 0.5 \leq X 2 \leq 20 ; 5 \leq$ $X 3 \leq 25 ; 2 \leq X 4 \leq 5$ for " $b$ ". The design of experiments is calculated with MSE criterion for 4 factors and 70 trial points. This design of experiment is also available on the web: http://www.mmd.rtu.lv.

So the 70 strength studies are calculated for each considered method. SW Simulation results (volume, maximal von Mises stress, axial displacement of the disk etc.) are entered into EDAOpt for approximation and subsequent global search.

Some optimization and approximation characteristics are shown in Table 4. Results of variants " $a$ " and " $b$ " are obtained with second order local polynomial approximation. Third order local polynomial approximation is used for variant " $\mathrm{c}$ ". Gaussian kernel coefficient $\alpha$ was varied for least value of crossvalidation error (4).

\begin{tabular}{|c|c|c|c|c|c|c|c|c|c|}
\hline \multirow{2}{*}{ Variant } & \multirow{2}{*}{$\alpha$} & $\begin{array}{c}\text { Approximation's } \\
\sigma_{\text {err }}[\%]\end{array}$ & \multicolumn{4}{|c|}{$\begin{array}{c}\text { Volume } v \\
{\left[\mathrm{~mm}^{3}\right]}\end{array}$} & \multicolumn{3}{|c|}{$\begin{array}{c}\text { Maximal von Mises stress } \\
\sigma_{\text {vonMises }}[\mathrm{MPa}]\end{array}$} \\
\cline { 3 - 11 } & & $\sigma_{\text {vonMises }}$ & $v$ & Predicted & Real & $\begin{array}{c}\text { Error } \\
{[\%]}\end{array}$ & Predicted & Real & $\begin{array}{c}\text { Error } \\
{[\%]}\end{array}$ \\
\hline $\mathrm{a}$ & 6 & 20.56 & 0.06 & 1003944 & 1003891 & 0.005 & 3.9999 & 3.833816 & 4.33 \\
\hline $\mathrm{b}$ & 3 & 40.28 & 2.03 & 923421 & 921740 & 0.018 & 3.9999 & 4.200354 & 4.77 \\
\hline $\mathrm{c}$ & 4 & 10.93 & 0.00 & 946180 & 946173 & 0.001 & 3.9998 & 4.125750 & 3.05 \\
\hline $\mathrm{d}$ & - & - & - & - & 1394900 & - & - & 3.3 & - \\
\hline
\end{tabular}

Table 4. Quantitative data of approximation and shape optimization of the ellipsoidal disk cross section.

The obtained metamodels are used for optimization of factors. The ellipsoidal disk volume is minimized by taking into account the specified constraints on displacement and stress level. The obtained shapes are presented in Fig. 12. As shown in table 4, the best results are obtained for variant " $b$ " (Fig. 13), where the volume is lower by $8.2 \%$ in comparison to variant "a" and on $2.6 \%$ - to " $c$ ". All 3 variants give significant advantage in volume (28.1 $33.9 \%$ ), comparing to variant " $\mathrm{d}$ "- the initial shape design with constant $10 \mathrm{~mm}$ thickness. 


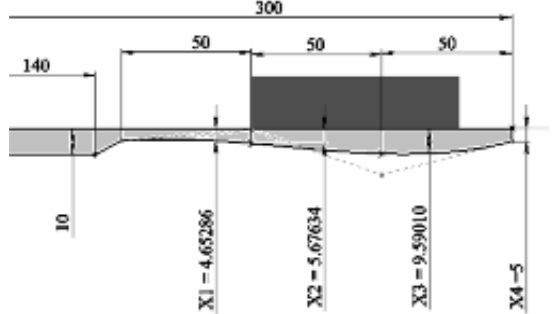

(a)

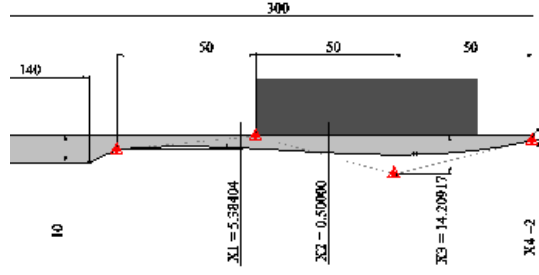

(b)

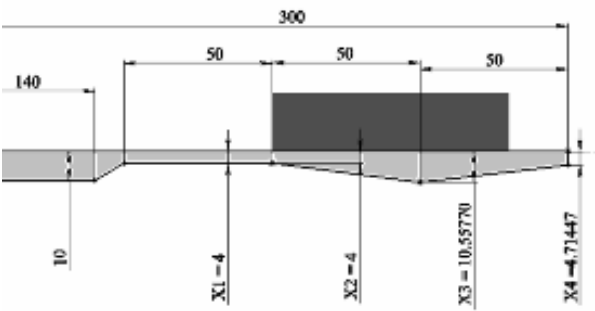

(c)

Fig. 12. Results of optimization of ellipsoidal disk. Shape of cross section is defined by (a) NURBS knot points, (b) NURBS polygon points, (c) points that are connected with straight lines.

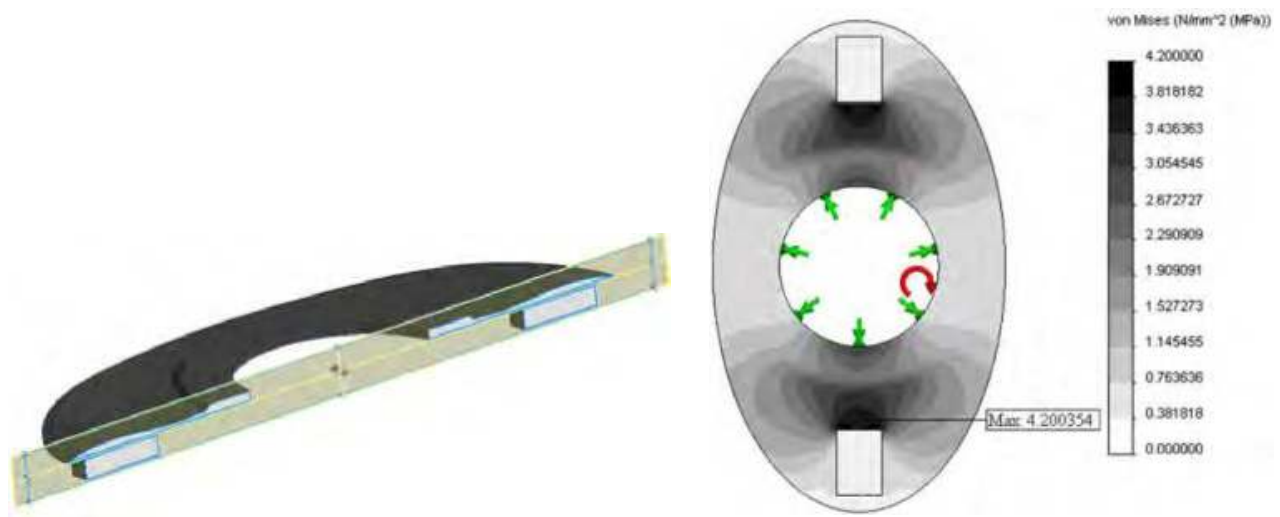

Fig. 13. Half of 3D model of optimal shape disk and real von Mises stresses distribution in it.

\subsection{Summary}

The proposed equipment allows using the standard wheel pair with removable measurement equipment as a tensometric wheel pair that considerably reduces material and time expenses required for preparing testing. By means of size and shape optimization, the total volume of the mounting disk of railway vehicle measurement system is reduced by $\sim 64$ $\%$ in comparison with the initial design. The method based on NURBS polygon points gives the shape with at least 3\% better objective (volume) than other used methods. 


\section{Automotive vehicle gage panel}

In this section designing the mechanical part of an automotive vehicle gage panel is discussed. Automotive vehicle gage panels (GP) must meet many requirements - such functional characteristics as appropriate stress levels under loads, eigenfrequencies, stiffnesses, weight, accuracy etc. and last but not least they must have minimal environmental pollution during service lifetime. The 3D geometrical models of the gage panel are elaborated using SW. Static and dynamic responses of the gage panel are calculated using SW Simulation and impacts to environment are evaluated using SW Sustainability that include such indices as total energy consumed, carbon footprint, air acidification and water eutrophication. The stationary and transient behaviors of the gage panel under dynamic excitation as well as stress distribution under static loading are investigated. Due to the complexity of the gage panel FEM models, the appropriate metamodels are elaborated based on design of experiments. These metamodels are used for multiobjective optimization using a global search procedure. Partial objectives are aggregated in the complex objective function for optimization purposes. Dynamic behavior of the gage panel is then verified by solution of the full FEM models in case of random vibrations.

\subsection{Specific requirements}

A constantly pressing problem is the development of safe and environmentally friendly engineering objects with high functional properties, attractive style and competitive price. We should try to take into account not only precisely measurable functional indices, but also such a difficult-to-formalize index as style of GP.

The Industrial Designer Society of America defines industrial design as the professional service of creating and developing concepts and specifications that optimize the function, value, and appearance of products and systems for the mutual benefit of both users and manufacturer. In fact, industrial designers focus their attention upon the form and user interaction of products. There are five critical goals (Ulrich \& Eppinger, 2008): 1) Utility: The product`s human interfaces should be safe, easy to use, and intuitive. Each feature should be shaped so that it communicates its function to the user. 2) Appearance: Form, line, proportion, and color are used to integrate the product into a pleasing whole. 3) Easy maintenance: Product must also be designed to communicate how they are to be maintained and repaired. 4) Low costs: Form and features have a large impact on tooling and production costs, so these must be considered jointly by the team. 5) Communication: Product design should communicate the corporate design philosophy and mission through the visual qualities of the products. The practical concept selection methods (Ulrich \& Eppinger, 2008) vary in their effectiveness and include the following: 1) External decision: Concepts are turned over to the customer, client, or some other external entity for selection. 2) Product champion: An influential member of the product development team chooses a concept based on personal preference. 3) Intuition: The concept is chosen by its feel. Explicit criteria or trade-offs are not used. The concept just seems better. 4) Multivoting: Each member of the team votes for several concepts. The concept with the most votes is selected. 5) Pros and cons: The team lists the strengths and weaknesses of each concept and makes a choice based upon group opinion. 6) Prototype and test: The organization builds and tests prototypes of each concept, making a selection based upon test data. 7) Decision matrices: 
The team rates each concept against pre specified selection criteria, which may be weighted. The concept selection method is built around the use of decision matrices for evaluating each concept with respect to a set of selection criteria. At the same time such formalized methods are elaborated as method of imprecision (Zimmermann, 2001) with noncompensating aggregation and compensating aggregation as well as fuzzy design method with different level interval algorithms.

The GP styles of different cars significantly differ and should be evaluated in context of the specific vehicle. At the same time style determines an arrangement of particular components (distances between gage axes etc.). In Fig. 14 we can see initial styles and the 3D model of the GP designed for new vehicles (Company Amoplant, 2011).
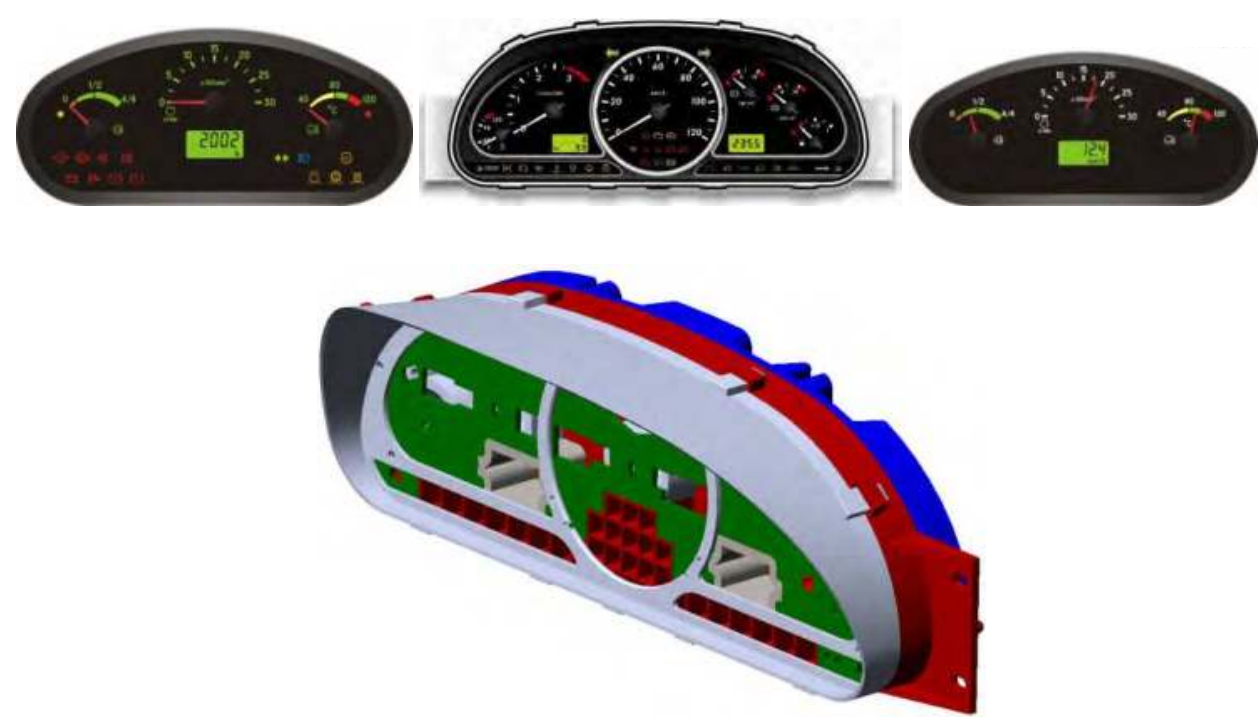

Fig. 14. Frontal view of GP of the initial styles and 3D geometrical model of GP.

\subsection{Vehicle GP optimization problem}

Now we can try to use the previously discussed approach for multiobjective shape optimization of the GP. The problem is stated as follows:

$$
\min _{x} F(x)=\left[F_{1}(x), F_{2}(x), \ldots, F_{k}(x)\right]^{T}
$$

subject to $g_{j}(x) \leq 0, j=1,2, \ldots, m$, and $h_{l}(x)=0, l=1,2, \ldots, e$;

where $k$ is the number of objective functions $F_{i}$;

$m$ is the number of inequality constraints;

$e$ is the number of equality constraints;

$x \in E^{n}$ is a vector of $n$ design variables.

First let us briefly discuss obtaining responses of GP for each particular objective. 


\subsection{Strength calculation of GP design}

Generally the strength of the GP design is checked on special vibrostands. The GP is subjected to different dynamic loads. Vibrostability and vibration strength of the GP are checked on excitations in the frequency domain from 10 to $250 \mathrm{~Hz}$. One of the main natural experiments is a test of shock resistance of the GP design under acceleration level $a=10 \mathrm{~g}$. Such experiments require significant material and time expenses and for optimization purposes the computer based design check must be used. The 3D geometrical model (Fig. 14) of the GP is created using SW and it consists of 18 parts: 6 deformable bodies and 12 rigid bodies that take into account the inertial characteristics of the internal devices. The deformable parts are made from the ABC 2020 plastic, but for the internal device bodies are assumed as alloy steel. The initial volume of the GP assembly is $v_{0}=764674 \mathrm{~cm}^{3}$ and mass $m_{0}=1.02 \mathrm{~kg}$. The 3D model of the GP assembly is used for FEM analysis by SW Simulation to evaluate different responses of the GP. The FE mesh (Fig. 15 ) is generated with curvature based mesh (max elements size $=9 \mathrm{~mm}$, min element size $=1.8 \mathrm{~mm}$, element size growth ratio $=1.5$ ), that ensures accurate discretization of the complex shape bodies of the GP. The FE mesh consists of $\sim 210,000$ nodes, $\sim 147,000$ elements, $\sim 640,000$ DOF.

In the initial design of the GP von Mises stresses from impact loading are shown on Fig. 15. We can see that maximal stresses are concentrated on the bracket's cross-section and it reaches $4 \mathrm{MPa}$. Other parts of the GP design are stressed considerably less. This implies that the bracket design should be improved.
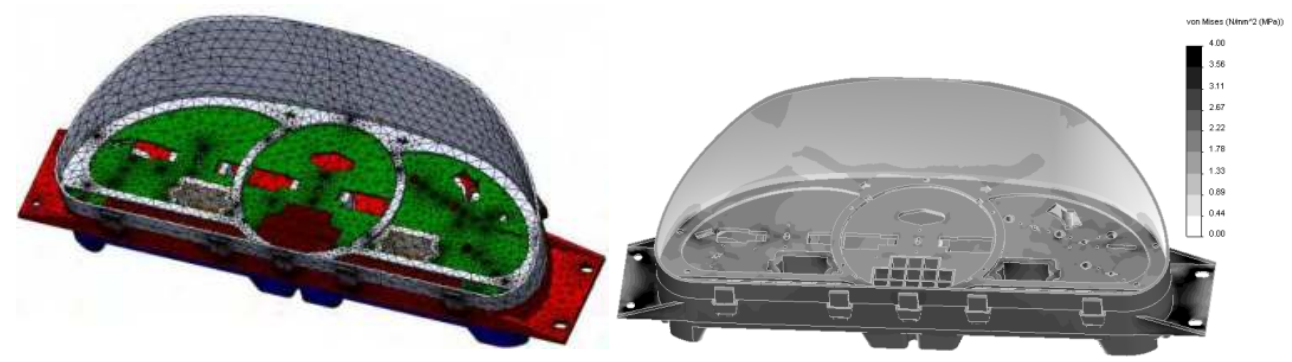

Fig. 15. Meshed 3D model and von Mises stresses distribution in initial design of GP.

\subsection{Frequency analysis of GP}

Frequency analysis is implemented to find natural frequencies of the GP model and evaluate possible resonance in the case of external excitation. The same FE mesh for model as considered before is used. The contacts between assembly's parts are defined as bonded. The numerical solver FFEPlus of SW is used for calculations.

The obtained results show that the fundamental frequency of the GP is sufficiently high $f_{1}=$ 170.47 Hz. The obtained mode shapes for the GP natural frequencies $\left(f_{2}=201.35 \mathrm{~Hz}, f_{3}=264\right.$ $\mathrm{Hz}, f_{4}=331.85 \mathrm{~Hz}$ ) are shown on Fig. 16. 


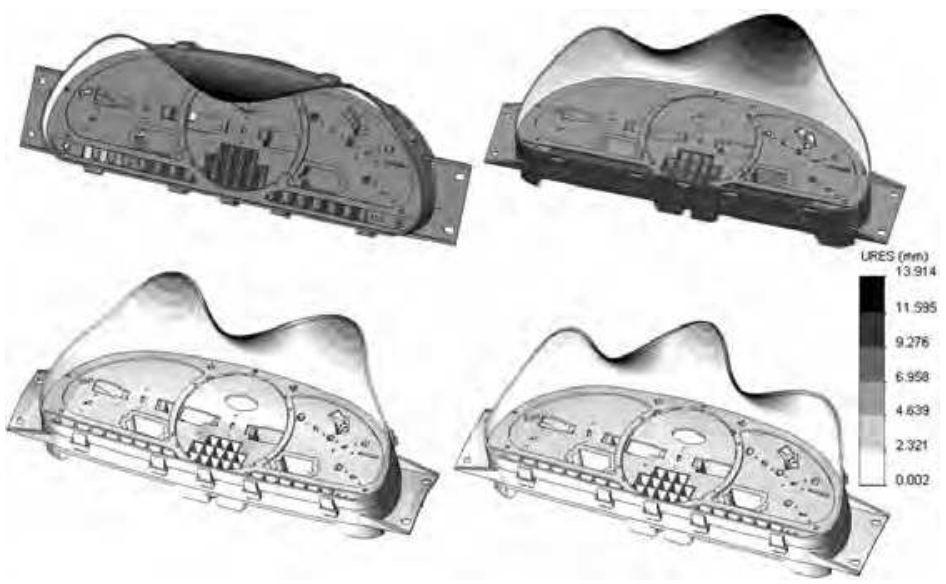

Fig. 16. The mode shapes of the four lower natural frequencies of GP.

\subsection{Sustainability analysis of GP}

SW Sustainability allows getting immediate feedback on the carbon footprint and other environmental impacts of the GP throughout its entire lifecycle, including material selection, production, transportation, use and end of life (Fig. 17).

\begin{tabular}{|c|c|c|c|c|c|c|}
\hline \multirow[t]{2}{*}{$\begin{array}{l}\text { Model } \\
\text { Name: }\end{array}$} & \multirow[t]{2}{*}{$\begin{array}{l}\text { Panelis } \\
\text { SW2.SLDPRT }\end{array}$} & \multirow[t]{2}{*}{ Material: } & \multirow[t]{2}{*}{ PVC Rigid } & $\begin{array}{l}\text { Volume: } \\
\text { Surface Area: }\end{array}$ & $\begin{array}{l}1.77 \mathrm{E}+5 \mathrm{~mm}^{3} \\
1.95 \mathrm{E}+5 \mathrm{~mm}^{2}\end{array}$ & \multirow[t]{2}{*}{$\begin{array}{l}\text { Manufacturing } \\
\text { Type: } \\
\text { Injection Molded }\end{array}$} \\
\hline & & & & Weight: & $230.47 \mathrm{~g}$ & \\
\hline
\end{tabular}

Environmental Impact
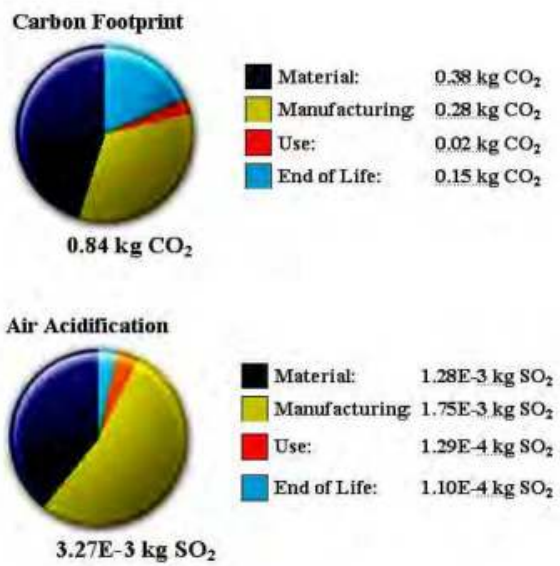

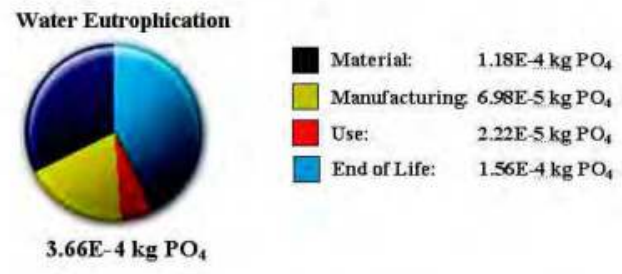

Total Energy Consumed

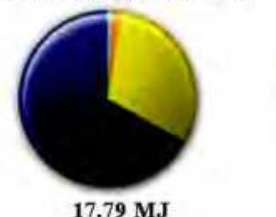

$\begin{array}{ll}\text { Material: } & 1190 \mathrm{M} \\ \text { Manufacturing } & 5.47 \mathrm{MJ} \\ \text { Use: } & 0.31 \mathrm{MJ} \\ \text { End of Life: } & 0.12 \mathrm{MJ}\end{array}$

Fig. 17. Environmental impact of the frame component of GP calculated by SW Sustainability. 


\subsection{Shape optimization of GP}

In this specific situation one of the solutions could be increasing the GP bracket cross-section thickness and changing the shape at the most stressed place. The cross-section shape for bracket's strengthening is defined by the 3 knot points (Fig. 18 a) of NURBS. Design parameters are coordinates of the knot points varied in the following ranges: $3 \leq X 1 \leq 6 ; 2 \leq$ $X 2 \leq 5 ; 0 \leq X 3 \leq 3$. As a cross-section profile is defined, the 3D-shape is created using the path curve (Fig. $18 \mathrm{~b}$ ). The same shape for strengthening is created on the second bracket of the GP frame component. A maximal von Mises stress in the bracket was minimized with constraint on the GP volume $\left(v<770500 \mathrm{~cm}^{3}\right)$.

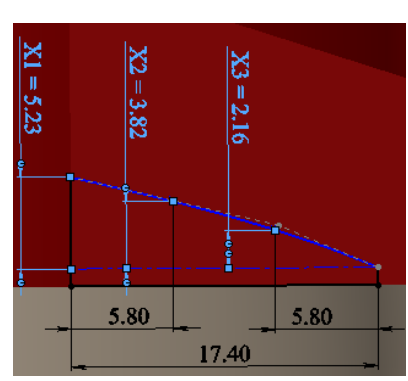

(a)

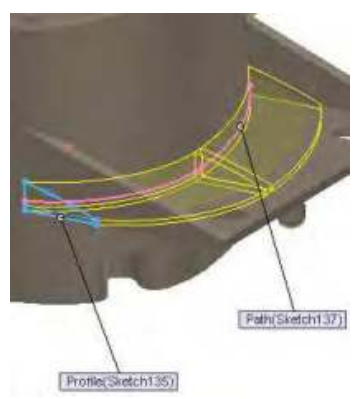

(b)

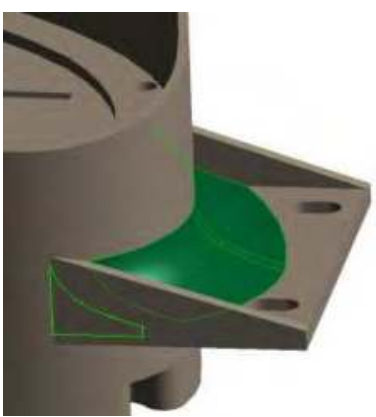

(c)

Fig. 18. Shape definition of bracket: (a) cross-section shape; (b) 3D- shape creation through path curve; (c) shape optimization result.

Von Mises stresses are compared in the design of the obtained shape and the initial shape (Fig. 19). There are 6 check points that show von Mises stresses distribution in the most stressed bracket cross-section. Volume of the obtained design is $v=770430 \mathrm{~cm}^{3}$. Change of

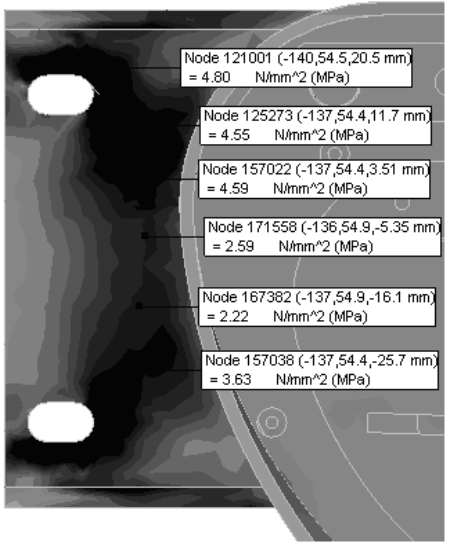

(a)

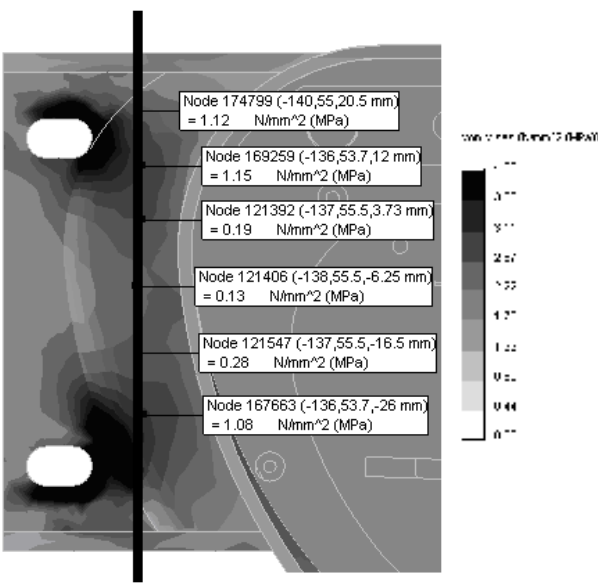

(b)

Fig. 19. Von Mises stress distribution in considered cross-section of: (a) initial design of GP and (b) optimized design of GP. 
the assembly volume is insignificant, but maximal von Mises stress level is reduced on $\sim 82.4$ $\%$. Von Mises stress level changes in the cross-section of the GP bracket for initial and optimized variants are presented in Fig. 20.

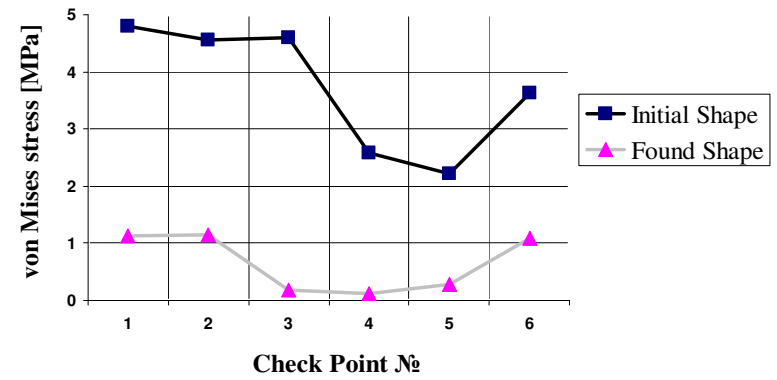

Fig. 20. Von Mises stress distribution in the bracket check points.

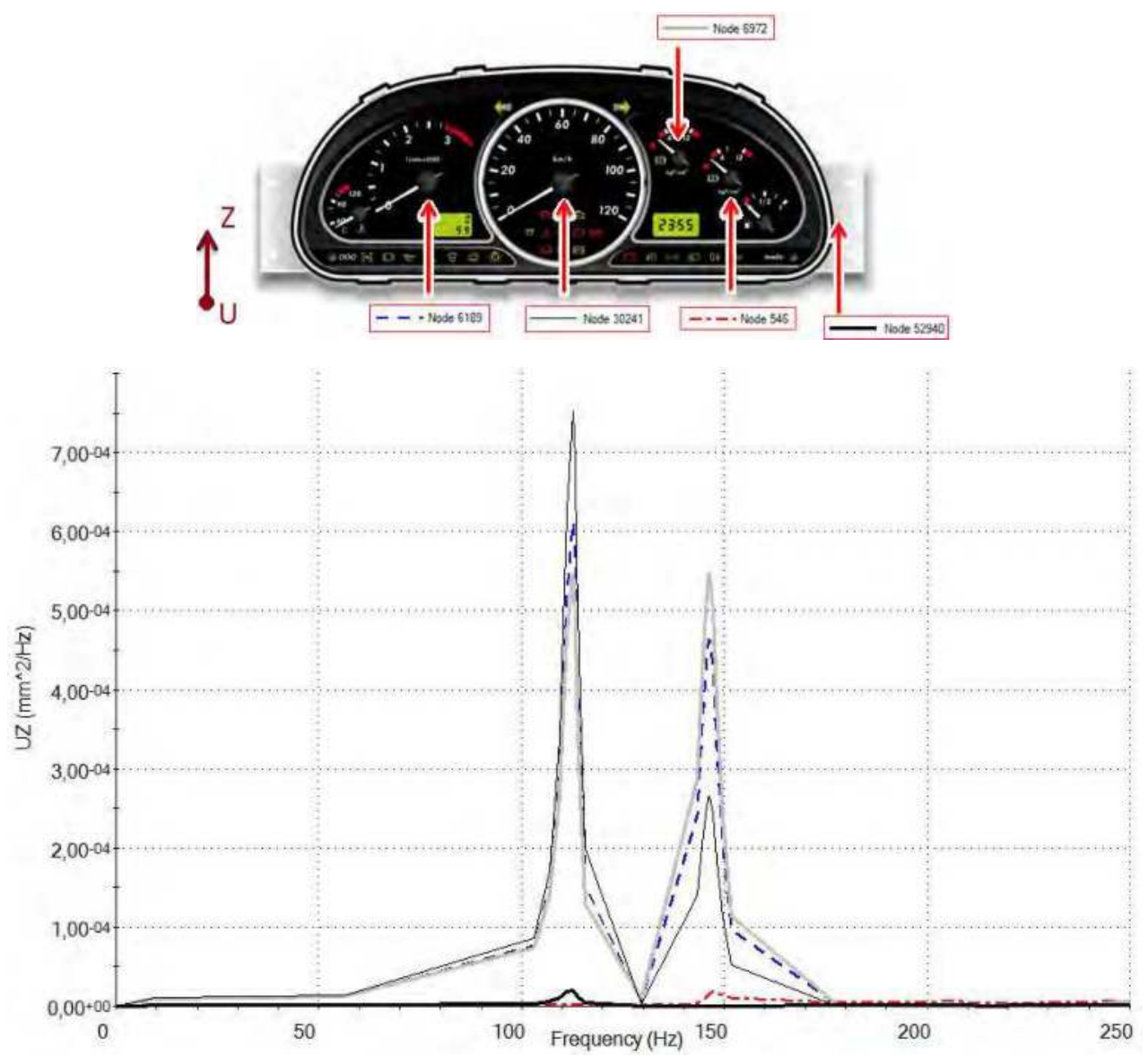

Fig. 21. PSD of vertical displacement in characteristic points of the GP. 
Of course, there are possibilities of further optimization of the path curve shape (Fig. 18b) and taking into account simultaneously the additional particular objectives such as maximal von Mises stress in material of the GP from dynamic loading in case of harmonic vibration excitations; styling of the GP using a-cut method (Zimmermann, 2001), the carbon footprint and other environmental impacts of the GP throughout its entire lifecycle calculated by SW Sustainability, as well as natural frequencies of the GP.

For the obtained optimal solution the dynamic behavior of the GP must be verified in case of the uniform base random excitations (see Fig. 21) by analysis of the full FEM model.

\section{Conclusion}

The results of shape optimization of the mechanical components for two different measurement systems are presented. The described approach allows obtaining smooth shapes that are easy to implement technologically. The jagged forms are excluded from the optimization process and there's no need for excessive computational resources. The most time-consuming step of the current approach is the FEM analysis of the full model for variants defined by design of experiments, the results of which are used for building metamodels of appropriate responses. Then the solution of various single objective problems and the implementation of different aggregation strategies for multiobjective optimization are relatively easy in order to obtain an acceptable final solution.

It will be interesting to compare the effectiveness of the current approach by using the metamodels obtained by kriging and radial basis functions instead of locally weighted polynomial approximations.

\section{Acknowledgment}

The research work reported here was made possible by partial financial support of Latvian Science Council grant No. 09.1267 and EU Project Filose (ID 231495).

\section{References}

Arora, J. S. (2004). Introduction to Optimum Design. - 2nd ed. - Elsevier

Audze, P. \& Eglajs, V. (1977). New Approach for Design of Experiments. Problems of Dynamics and Strength, Vol. 35, Riga: Zinatne, RU, pp. 104-107

Auzins, J. Direct Optimization of Experimental Designs. (2004). 10th AIAA/ISSMO Multidisciplinary Analysis and Optimization Conference, Albany, NY, 28 Aug.-2 Sep. 2004., AIAA Paper 2004-4578, CD-ROM Number 17, pp. 1-17

Auzins, J. \& Janushevskis, A. (2007). Design of Experiments and Analysis. Riga, LV

Auzins, J. \& Janushevskis, A. (2002). New Experimental Designs for Metamodelling and Optimization, Proceedings of the Fifth World Congress on Computational Mechanics (WCCM V), July 7-12, 2002, Vienna, Austria, Editors: Mang, H.A.; Rammerstorfer, F.G.; Eberhardsteiner, J., Publisher: Vienna University of Technology, Austria, ISBN 3-9501554-0-6, pp. 1-10

Auzins, J.; Janushevskis, J.; Janushevskis, A. \& Kalnins, K. (2006). Optimisation of designs for natural and numerical experiments, Extended Abstracts of the 6th Int ASMOUK/ISSMO conference on Engineering Design Optimization. Oxford, UK, pp. 118 - 121. 
Bendsoe, M. P. \& Sigmund, O. (2003). Topology Optimization: Theory, Methods and Application. -2nd ed. - Heidelberg (Berlin): Springer

Company Amoplant (2011). Available from http://www.amoplant.lv

Forrester, A. I. J.; Sóbester, A. \& Keane, A.J. (2008). Engineering Design via Surrogate Modelling. A Practical Guide. Wiley

Grigorov, I. N. (2004). Transmitting Magnetic Loop Antennas. Moscow: RadioSoft. RU.

Hart, T. (1986). W5QJR. The Loop. Small High Efficiency Antennas. Melbourne: Sage-American Co.

Janushevskis, A.; Akinfiev; T., Auzins, J. \& Boyko, A. (2004). A Comparative Analysis of Global Search Procedures. Proc. Estonian Acad. Sci. Eng., Vol.10, No.4, pp. 235-250

Janushevskis, A. \& Melnikovs, A. (2010). Shape Optimization of Block. Scientific Jour. of RTU: Transport and Engineering. Mechanics. Series 6. Vol. 33, Riga, pp. 89-97

Janushevskis, A.; Melnikovs, A. \& Boyko, A. (2010). Shape Optimization of Mounting Disk of Railway Vehicle Measurement System, Jour. of Vibroengineering, Vol. 12, Issue 4, pp. $436-443$

Liang Q.Q.; Xie Y.M. \& Steven G.P. (2001) A Performance Index for Topology and Shape Optimization of Plate Bending Problems with Displacement Constraints. Struct. and Multidisciplinary Optimization, Berlin, pp. 393-399

Lombard M. (2009). SolidWorks 2009 Bible. Indianapolis: Wiley

McKay, M.D.; Beckman, R.J. \& Conover, W.J. (1979). A comparison of three methods for selecting values of input variables in the analysis of output from computer code. Technometrics 21, pp. 239-245

Mullerschon, H.; Lazarov, N. \& Witowski, K. (2010). Application of Topology Optimization for Crash with LC-OPT/Topology. Proc. 11th Int LS-DYNA Users Conference, pp. 17$39-17-46$

Papadrakakis, M.; Lagaros, N.; Thierauf, G. \& Cai, J. (1998). Advanced solution methods in structural optimisation using evolution strategies, Eng. Comp. Jour., 15 (1), pp. 12-34

Sacks, J.; Welch, W.J.; Mitchell, T.J. \& Wynn, H.P. (1989). Designs and analysis of computer experiments, Statist. Sci., 4, pp. 409-435

Saxena, A. \& Sahay, B. (2005). Computer aided engineering design. India: Anamaya

Schmit, L. A. \& Farcshi, B. (1971). Some Approximation Concepts for Structural Synthesis. AIAAJ.,Vol.12, No. 5, pp. 692-699

Sladkowsky, A. \& Pogorelov, D. (2008). Investigation of Dynamical Interactions at Rail Wheel Contact in Case of Flat of Wheel. Bulletin of Ukraine National University, No. 5, RU pp. 88-95

State Railway Research Institute. (1998). Codes of Design of Railway Wagons with Wheel Span $1420 \mathrm{~mm}$. Moscow, RU

Ulrich, K.T. \& Eppinger, S. D. (2008). Product Design and Development. McGraw - Hill Int.

Vanderplaats, G. N. (2004). Numerical Optimization Techniques for Engineering Design with Applications. 4th Ed., Vanderplaats Research \& Development, Inc. Colorado Springs, USA

Zimmermann, H. (2001). Fuzzy Set Theory and its Applications. Boston: Kluwer Acad. Publ. 


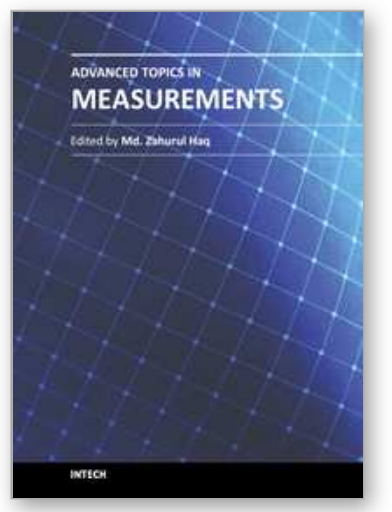

\author{
Advanced Topics in Measurements \\ Edited by Prof. Zahurul Haq
}

ISBN 978-953-51-0128-4

Hard cover, 400 pages

Publisher InTech

Published online 07, March, 2012

Published in print edition March, 2012

Measurement is a multidisciplinary experimental science. Measurement systems synergistically blend science, engineering and statistical methods to provide fundamental data for research, design and development, control of processes and operations, and facilitate safe and economic performance of systems. In recent years, measuring techniques have expanded rapidly and gained maturity, through extensive research activities and hardware advancements. With individual chapters authored by eminent professionals in their respective topics, Advanced Topics in Measurements attempts to provide a comprehensive presentation and in-depth guidance on some of the key applied and advanced topics in measurements for scientists, engineers and educators.

\title{
How to reference
}

In order to correctly reference this scholarly work, feel free to copy and paste the following:

Alexander Janushevskis, Janis Auzins, Anatoly Melnikovs and Anita Gerina-Ancane (2012). Shape Optimization of Mechanical Components for Measurement Systems, Advanced Topics in Measurements, Prof. Zahurul Haq (Ed.), ISBN: 978-953-51-0128-4, InTech, Available from: http://www.intechopen.com/books/advanced-topics-in-measurements/shape-optimization-of-mechanicalcomponents-for-measurement-systems

\section{INTECH}

open science | open minds

\author{
InTech Europe \\ University Campus STeP Ri \\ Slavka Krautzeka 83/A \\ 51000 Rijeka, Croatia \\ Phone: +385 (51) 770447 \\ Fax: +385 (51) 686166 \\ www.intechopen.com
}

\author{
InTech China \\ Unit 405, Office Block, Hotel Equatorial Shanghai \\ No.65, Yan An Road (West), Shanghai, 200040, China \\ 中国上海市延安西路65号上海国际贵都大饭店办公楼405单元 \\ Phone: +86-21-62489820 \\ Fax: +86-21-62489821
}


(C) 2012 The Author(s). Licensee IntechOpen. This is an open access article distributed under the terms of the Creative Commons Attribution 3.0 License, which permits unrestricted use, distribution, and reproduction in any medium, provided the original work is properly cited. 